\title{
Predicate Abstraction via Symbolic Decision Procedures
}

\author{
Shuvendu K. Lahiri, Thomas Ball, and Byron Cook \\ Microsoft Research \\ \{shuvendu, tball, bycook\}@microsoft.com
}

\begin{abstract}
We present a new approach for performing predicate abstraction based on symbolic decision procedures. A symbolic decision procedure for a theory $T\left(S D P_{T}\right)$ takes sets of predicates $G$ and $E$ and symbolically executes a decision procedure for $T$ on $G^{\prime} \cup\{\neg e \mid e \in E\}$, for all the subsets $G^{\prime}$ of $G$. The result of $S D P_{T}$ is a shared expression (represented by a directed acyclic graph) that implicitly represents the answer to a predicate abstraction query.

We present symbolic decision procedures for the logic of Equality and Uninterpreted Functions(EUF) and Difference logic (DIF) and show that these procedures run in pseudo-polynomial (rather than exponential) time. We then provide a method to construct $S D P$ 's for simple mixed theories (including EUF + DIF) using an extension of the Nelson-Oppen combination method. We present preliminary evaluation of our procedure on predicate abstraction benchmarks from device driver verification in SLAM.
\end{abstract}

\section{Introduction}

Predicate abstraction is a technique for automatically creating finite abstract models of finite and infinite state systems [10. The method has been widely used in abstracting finite-state models of programs in SLAM 2 and numerous other software verification projects 11,4. It has also been used for synthesizing loop invariants 9 , and verifying distributed protocols [8, 13.

The fundamental operation in predicate abstraction can be summarized as follows: Given a set of predicates $P$ describing some set of properties of the system state, and a formula $e$, compute the weakest Boolean formula $\mathcal{F}_{P}(e)$ over the predicates $P$ that implies 11 . Most implementations of predicate abstraction 10,2 construct $\mathcal{F}_{P}(e)$ by collecting the set of cubes (a conjunction of the predicates or their negations) over $P$ that imply $e$. The implication is checked using a first-order theorem prover. This method may require making a very large $\left(2^{|P|}\right.$ in the worst case) number of calls to a theorem prover and can be expensive.

\footnotetext{
${ }^{1}$ The dual of this problem, which is to compute the strongest Boolean formula $\mathcal{G}_{P}(e)$ that is implied by $e$, can be expressed as $\neg \mathcal{F}_{P}(\neg e)$.
} 
Several techniques have been suggested to improve the performance of predicate abstraction. Some techniques enumerate the cubes over $P$ in an increasing order of size [8, 9, 18. However, these techniques still require an exponential number of theorem prover calls in the worst case, and demonstrate worst case behavior in practice. Other techniques sacrifice precision to gain efficiency, by only considering cubes of some fixed length [2].

Alternately, predicate abstraction can be formulated as a quantifier elimination problem. Lahiri et al. 13] and Clarke et al. 5] perform predicate abstraction by reducing the problem to Boolean quantifier elimination. The former method first transforms a first-order quantifier elimination problem into Boolean quantifier elimination by encoding first-order formulas into Boolean formulas; the latter assumes a finite representation of integers. The method in 13 first converts the quantifier-free first-order formula to a Boolean formula such that the translation preserves the set of satisfying assignments of the Boolean variables in the original variable. Both these techniques use incremental Boolean Satisfiability (SAT) techniques [5, 14] to perform the Boolean quantifier elimination. Namjoshi and Kurshan [15] also proposed using quantifier elimination for firstorder logic directly to perform predicate abstraction - however many theories (such as the theory of Equality with Uninterpreted Functions) do not admit quantifier elimination.

Most of the above approaches use decision procedures or SAT solvers as "black boxes", at best in an incremental fashion, to perform predicate abstraction. We believe that having a customized procedure for predicate abstraction can help improve the efficiency of predicate abstraction on large problems.

We propose a new way to perform predicate abstraction based on symbolic decision procedures. A symbolic decision procedure for a theory $T\left(S D P_{T}\right)$ takes sets of predicates $G$ and $E$ and symbolically executes a decision procedure for $T$ on $G^{\prime} \cup\{\neg e \mid e \in E\}$, for all the subsets $G^{\prime}$ of $G$. The output of $S D P_{T}(G, E)$ is a shared expression (an expression where common subexpressions can be shared) representing those subsets $G^{\prime} \subseteq G$, for which $G^{\prime} \cup\{\neg e \mid e \in E\}$ is unsatisfiable. We show that such a procedure can be used to compute $\mathcal{F}_{P}(e)$ for performing predicate abstraction.

We present symbolic decision procedures for the logic of Equality and Uninterpreted Functions(EUF) and Difference logic (DIF) and show that these procedures run in polynomial and pseudo-polynomial time respectively, and therefore produce compact shared expressions. We provide a method to construct $S D P$ for a combination of two simple theories $T_{1} \cup T_{2}$ (including EUF + DIF), by using an extension of the Nelson-Oppen combination method. We use Binary Decision Diagrams (BDDs) 3 to construct $\mathcal{F}_{P}(e)$ from the shared representations efficiently in practice. The proofs for the theorems and lemmas can be found in a detailed technical report [12.

We present a preliminary evaluation of our procedure on predicate abstraction benchmarks from device driver verification in SLAM, and show that our method outperforms existing methods for doing predicate abstraction. 


\section{Setup}

Figure 1 defines the syntax of a quantifier-free fragment of first-order logic. An expression in the logic can either be a term or a formula. A term can either be a variable or an application of a function symbol to a list of terms. A formula can be the constants true or false or an atomic formula or Boolean combination of other formulas. Atomic formulas can be formed by an equality between terms or by an application of a predicate symbol to a list of terms.

$$
\begin{aligned}
\text { term }: & :=\text { variable } \mid \text { function-symbol }(\text { term }, \ldots, \text { term }) \\
\text { formula }::=\text { true } \mid \text { false } \mid \text { atomic-formula } & \\
\mid & \text { formula } \wedge \text { formula } \mid \text { formula } \vee \text { formula } \mid \neg \text { formula } \\
\text { atomic-formula }: & =\text { term }=\text { term } \mid \text { predicate-symbol }(\text { term }, \ldots, \text { term })
\end{aligned}
$$

Fig. 1. Syntax of a quantifier-free fragment of first-order logic

The function and predicate symbols can either be uninterpreted or can be defined by a particular theory. For instance, the theory of integer linear arithmetic defines the function-symbol "+" to be the addition function over integers and " $<$ " to be the comparison predicate over integers. If an expression involves function or predicate symbols from multiple theories, then it is said to be an expression over mixed theories.

A formula $F$ is said to be satisfiable if it is possible to assign values to the various symbols in the formula from the domains associated with the theories to make the formula true. A formula is valid if $\neg F$ is not satisfiable (or unsatisfiable). We say a formula $A$ implies a formula $B(A \Rightarrow B)$ if and only if $(\neg A) \vee B$ is valid.

We define a shared expression to be a Directed Acyclic Graph (DAG) representation of an expression where common subexpressions can be shared, by using names to refer to common subexpressions. For example, the intermediate variable $t$ refers to the expression $e_{1}$ in the shared expression "let $t=$ $e_{1}$ in $\left(e_{2} \wedge t\right) \vee\left(e_{3} \wedge \neg t\right) "$.

\subsection{Predicate Abstraction}

A predicate is an atomic formula or its negation 2 . If $G$ is a set of predicates, then we define $\widetilde{G} \doteq\{\neg g \mid g \in G\}$, to be the set containing the negations of the predicates in $G$. We use the term "predicate" in a general sense to refer to any atomic formula or its negation and should not be confused to only mean the set of predicates that are used in predicate abstraction.

Definition 1. For a set of predicates $P$, a literal $l_{i}$ over $P$ is either a predicate $p_{i}$ or $\neg p_{i}$, where $p_{i} \in P$. A cube $c$ over $P$ is a conjunction of literals. A clause

\footnotetext{
${ }^{2}$ We always use the term "predicate symbol" (and not "predicate") to refer to symbols like " $<"$ "
} 
cl over $P$ is a disjunction of literals. Finally, a minterm over $P$ is a cube with $|P|$ literals, and exactly one of $p_{i}$ or $\neg p_{i}$ is present in the cube.

Given a set of predicates $P \doteq\left\{p_{1}, \ldots, p_{n}\right\}$ and a formula $e$, the main operation in predicate abstraction involves constructing the weakest Boolean formula $\mathcal{F}_{P}(e)$ over $P$ such that $\mathcal{F}_{P}(e) \Rightarrow e$. The expression $\mathcal{F}_{P}(e)$ can be expressed as the set of all the minterms over $P$ that imply $e$ :

$$
\mathcal{F}_{P}(e)=\bigvee\{c \mid c \text { is a minterm over } P \text { and } c \text { implies } e\}
$$

Proposition 1. For a set of predicates $P$ and a formula e, (i) $\mathcal{F}_{P}(\neg e) \Rightarrow$ $\neg \mathcal{F}_{P}(e)$, (ii) $\mathcal{F}_{P}\left(e_{1} \wedge e_{2}\right) \Leftrightarrow \mathcal{F}_{P}\left(e_{1}\right) \wedge \mathcal{F}_{P}\left(e_{2}\right)$, and (iii) $\mathcal{F}_{P}\left(e_{1}\right) \vee \mathcal{F}_{P}\left(e_{2}\right) \Rightarrow$ $\mathcal{F}_{P}\left(e_{1} \vee e_{2}\right)$ (refer to [12] for proofs).

The operation $\mathcal{F}_{P}(e)$ does not distribute over disjunctions. Consider the example where $P \doteq\{x \neq 5\}$ and $e \doteq x<5 \vee x>5$. In this case, $\mathcal{F}_{P}(e)=$ $x \neq 5$. However $\mathcal{F}_{P}(x<5)=$ false and $\mathcal{F}_{P}(x>5)=$ false and thus $\left(\mathcal{F}_{P}(x<5) \vee \mathcal{F}_{P}(x>5)\right)$ is not the same as $\mathcal{F}_{P}(e)$.

The above properties suggest that one can adopt a two-tier approach to compute $\mathcal{F}_{P}(e)$ for any formula $e$ :

1. Convert $e$ into an equivalent Conjunctive Normal Form (CNF), which comprises of a conjunction of clauses, i.e., $e \equiv\left(\bigwedge_{i} c l_{i}\right)$.

2. For each clause $c l_{i} \doteq\left(e_{1}^{i} \vee e_{2}^{i} \ldots \vee e_{m}^{i}\right)$, compute $r_{i} \doteq \mathcal{F}_{P}\left(c l_{i}\right)$ and return $\mathcal{F}_{P}(e) \doteq \bigwedge_{i} r_{i}$.

We focus here on computing $\mathcal{F}_{P}\left(\bigvee_{e_{i} \in E} e_{i}\right)$ when $e_{i}$ is a predicate. Unless specified otherwise, we always use $e$ to denote $\left(\bigvee_{e_{i} \in E} e_{i}\right)$, a disjunction of predicates in the set $E$ in the sequel. For converting a formula to an equivalent $\mathrm{CNF}$ efficiently, we can use the method proposed by McMillan [14.

\section{Symbolic Decision Procedures (SDP)}

We now show how to perform predicate abstraction using symbolic decision procedures. We start by describing a saturation-based decision procedure for a theory $T$ and then use it to describe the meaning of a symbolic decision procedure for the theory $T$. Finally, we show how a symbolic decision procedure can yield a shared expression of $\mathcal{F}_{P}(e)$ for predicate abstraction.

$$
\begin{gathered}
\frac{X=Y}{Y=X} \\
\frac{Y \quad Y=Z}{X=Z}
\end{gathered}
$$

$$
\begin{gathered}
\frac{X=Y \quad X \neq Y}{\perp} \\
X_{1}=Y_{1} \quad \cdots \quad X_{n}=Y_{n} \\
f\left(X_{1}, \cdots, X_{n}\right)=f\left(Y_{1}, \cdots, Y_{n}\right)
\end{gathered}
$$

Fig. 2. Inference rules for theory of equality and uninterpreted functions 
A set of predicates $G$ (over theory $T$ ) is unsatisfiable if the formula $\left(\bigwedge_{g \in G} g\right.$ ) is unsatisfiable. For a given theory $T$, the decision procedure for $T$ takes a set of predicates $G$ in the theory and checks if $G$ is unsatisfiable. A theory is defined by a set of inference rules. An inference rule $R$ is of the form:

$\frac{A_{1} \quad A_{2} \quad \cdots \quad A_{n}}{A}$

which denotes that the predicate $A$ can be derived from predicates $A_{1}, \ldots, A_{n}$ in one step. Each theory has least one inference rule for deriving contradiction $(\perp)$. We also use $g:-g_{1}, \ldots, g_{k}$ to denote that the predicate $g$ (or $\perp$, where $g=\perp$ ) can be derived from the predicates $g_{1}, \ldots, g_{k}$ using one of the inference rules in a single step. Figure 2 describes the inference rules for the theory of Equality and Uninterpreted Functions.

\subsection{Saturation Based Decision Procedures}

Consider a simple saturation-based procedure $D P_{T}$ shown in Figure 3 , that takes a set of predicates $G$ as input and returns SATISFIABLE or UNSATISFIABLE.

The algorithm maintains two sets: (i) $W$ is the set of predicates derived from $G$ up to (and including) the current iteration of the loop in step (2); (ii) $W^{\prime}$ is the set of all predicates derived before the current iteration. These sets are initialized in step (1). During each iteration of step (2), if a new predicate $g$ can be derived from a set of predicates $\left\{g_{1}, \ldots, g_{k}\right\} \subseteq W^{\prime}$, then $g$ is added to $W$. The loop terminates after a bound $\operatorname{derivDepth}_{T}(G)$. In step $(3)$, we check if any subset of facts in $W$ can derive contradiction. If such a subset exists, the algorithm returns UNSATISFIABLE, otherwise it returns SATISFIABLE.

The parameter $d \doteq \operatorname{derivDepth}_{T}(G)$ is a bound (that is determined solely by the set $G$ for the theory $T$ ) such that if the loop in step (2) is repeated for at least $d$ steps, then $D P_{T}(G)$ returns UnSATISFIABLE if and only if $G$ is unsatisfiable. If such a bound exists for any set of predicates $G$ in the theory, then $D P_{T}$ procedure implements a decision procedure for $T$.

Definition 2. A theory $T$ is called a saturation theory, if the procedure $D P_{T}$ described in Figure 3 implements a decision procedure for $T$.

In the rest of the paper, we only consider saturation theories. To show that a theory $T$ is a saturation theory, it suffices to consider a decision procedure algorithm for $T$ (say $A_{T}$ ) and show that $D P_{T}$ implements $A_{T}$. This can be shown by deriving a bound on $\operatorname{derivDepth}_{T}(G)$ for any set $G$ in the theory.

\subsection{Symbolic Decision Procedure}

For a (saturation) theory $T$, a symbolic decision procedure for $T\left(S D P_{T}\right)$ takes sets of predicates $G$ and $E$ as inputs, and symbolically simulates $D P_{T}$ on $G^{\prime} \cup \widetilde{E}$, for every subset $G^{\prime} \subseteq G$. The output of $S_{D P_{T}}(G, E)$ is a symbolic expression representing those subsets $G^{\prime} \subseteq G$, such that $G^{\prime} \cup \widetilde{E}$ is unsatisfiable. Thus with $|G|=n$, a single run of $S D P_{T}$ symbolically executes $2^{n}$ runs of $D P_{T}$. 
1. Initialize $W \leftarrow G$. $W^{\prime} \leftarrow\{\}$.

2. For $i=1$ to $\operatorname{derivDepth}_{T}(G)$ :

(a) Let $W^{\prime} \leftarrow W$.

(b) For every fact $g \notin W^{\prime}$, if $\left(g:-g_{1}, \ldots, g_{k}\right)$ and $g_{m} \in W^{\prime}$ for all $m \in[1, k]$ :

$-W \leftarrow W \cup\{g\}$.

3. If $\left(\perp:-g_{1}, \ldots, g_{k}\right)$ and $g_{m} \in W$ for all $m \in[1, k]$ :

- return UNSATISFIABLE

4. else return SATISFIABLE

Fig. 3. $D P_{T}(G)$ : A simple saturation-based procedure for theory $T$

We introduce a set of Boolean variables $B_{G} \doteq\left\{b_{g} \mid g \in G\right\}$, one for each predicate in $G$. An assignment $\sigma: B_{G} \rightarrow\{$ true, false $\}$ over $B_{G}$ uniquely represents a subset $G^{\prime} \doteq\left\{g \mid \sigma\left(b_{g}\right)=\right.$ true $\}$ of $G$.

Figure 4 presents the symbolic decision procedure for a theory $T$, which symbolically executes the saturation based decision procedure $D P_{T}$ on all possible subsets of the input component $G$. Just like the $D P_{T}$ algorithm, this procedure also has three main components: initialization, saturation and contradiction detection. The algorithm also maintains sets $W$ and $W^{\prime}$, as the $D P_{T}$ algorithm does.

Since $\operatorname{SDP}(G, E)$ has to execute $D P_{T}\left(G^{\prime} \cup \widetilde{E}\right)$ on all $G^{\prime} \subseteq G$, the number of steps to iterate the saturation loop equals the maximum $\operatorname{derivDepth}_{T}\left(G^{\prime} \cup \widetilde{E}\right)$ for any $G^{\prime} \subseteq G$. For a set of predicates $S$, we define the bound $\operatorname{maxDerivDepth}_{T}(S)$ as follows:

$$
\operatorname{maxDerivDepth}_{T}(S) \doteq \max \left\{\operatorname{derivDepth}_{T}\left(S^{\prime}\right) \mid S^{\prime} \subseteq S\right\}
$$

During the execution, the algorithm constructs a set of shared expressions with the variables over $B_{G}$ as the leaves and temporary variables $t[\cdot]$ to name intermediate expressions. We use $t[(g, i)]$ to denote the expression for the predicate $g$ after the iteration $i$ of the loop in step (2) of the algorithm. We use $t[(g, \top)]$ to denote the top-most expression for $g$ in the shared expression. Below, we briefly describe each of the phases of $S D P_{T}$ :

Initialization [Step (1)]. The set $W$ is initialized to $G \cup \widetilde{E}$ and $W^{\prime}$ to \{\} . The leaves of the shared expression symbolically encode each subset $G^{\prime} \cup \widetilde{E}$, for every $G^{\prime} \subseteq G$. For each $g \in G$, the leaf $t[(g, 0)]$ is set to $b_{g}$. For any $e_{i} \in E$, since $\neg e_{i}$ is present in all possible subset $G^{\prime} \cup \widetilde{E}$, we replace the leaf for $\neg e_{i}$ with true.

Saturation [Step (2)]. For each predicate $g, S(g)$ is the set of derivations of $g$ from predicates in $W^{\prime}$ during any iteration. For any predicate $g$, we first add all the ways to derive $g$ until the previous steps by adding $t[(g, i-1)]$ to $S(g)$. Every time $g$ can be derived from some set of facts $g_{1}, \ldots, g_{k}$ such that each $g_{j}$ is in $W^{\prime}$, we add this derivation to $S(g)$ in Equation 2 At the end of the iteration $i, t[(g, i)]$ and $t[(g, \top)]$ are updated with the set of derivations in $S(g)$. The loop is executed maxDerivDepth $\operatorname{mov}_{T}(G \cup \widetilde{E})$ times.

Contradiction [Steps $(3,4)$ ]. We know that if $G^{\prime} \cup \widetilde{E}$ is unsatisfiable, then $G^{\prime}$ implies $e$ (recall, $e$ stands for $\bigvee_{e_{i} \in E} e_{i}$ ). Therefore, each derivation of $\perp$ 
1. Initialization

(a) $W \leftarrow G \cup \widetilde{E}$ and $W^{\prime} \leftarrow\{\}$.

(b) For each $g \in G, t[(g, 0)] \leftarrow b_{g}$.

(c) For each $e_{i} \in E, t\left[\left(\neg e_{i}, 0\right)\right] \leftarrow$ true.

2. For $i=1$ to $\operatorname{maxDerivDepth}_{T}(G \cup \widetilde{E})$ do:

(a) $W^{\prime} \leftarrow W$.

(b) Initialize $S(g)=\{\}$, for any predicate $g$.

(c) For every $g \in W^{\prime}, S(g) \leftarrow S(g) \cup\{t[(g, i-1)]\}$.

(d) For every $g$, if $\left(g:-g_{1}, \ldots, g_{k}\right)$ and $g_{m} \in W^{\prime}$ for all $m \in[1, k]$ :

i. Update the set of derivations of $g$ at this level:

$$
S(g) \leftarrow S(g) \cup\left\{\left(\bigwedge_{m \in[1, k]} t\left[\left(g_{m}, i-1\right)\right]\right)\right\}
$$

ii. $W \leftarrow W \cup\{g\}$.

(e) For each $g \in W: t[(g, i)] \leftarrow \bigvee_{d \in S(g)} d$

(f) For each $g \in W, t[(g, \top)] \leftarrow t[(g, i)]$

3. Check for contradiction:

(a) Initialize $S(e)=\{\}$.

(b) For every $\left\{g_{1}, \ldots, g_{k}\right\} \subseteq W$, if $\left(\perp:-g_{1}, \ldots, g_{k}\right)$ then

$$
S(e) \leftarrow S(e) \cup\left\{\left(\bigwedge_{m \in[1, k]} t\left[\left(g_{m}, \top\right)\right]\right)\right\}
$$

(c) Create the derivations for the goal $e$ as $t[e] \leftarrow\left(\bigvee_{d \in S(e)} d\right)$

4. Return the shared expression for $t[e]$.

Fig. 4. Symbolic decision procedure $S D P_{T}(G, E)$ for theory $T$. The expression $e$ stands for $\bigvee_{e_{i} \in E} e_{i}$.

from predicates in $W$ gives a new derivation of $e$. The set $S(e)$ collects these derivations and constructs the final expression $t[e]$, which is returned in step (4).

The output of the procedure is the shared expression $t[e]$. The leaves of the expression are the variables in $B_{G}$. The only operations in $t[e]$ are conjunction and disjunction; $t[e]$ is thus a Boolean expression over $B_{G}$. We now define the evaluation of a (shared) expression with respect to a subset $G^{\prime} \subseteq G$.

Definition 3. For any expression $t[x]$ whose leaves are in set $B_{G}$, and a set $G^{\prime} \subseteq G$, we define eval $\left(t[x], G^{\prime}\right)$ as the evaluation of $t[x]$, after replacing each leaf $b_{g}$ of $t[x]$ with true if $g \in G^{\prime}$ and with false otherwise.

The following theorem explains the correctness of the symbolic decision procedure.

Theorem 1. If $t[e] \doteq S D P_{T}(G, E)$, then for any set of predicates $G^{\prime} \subseteq G$, $\operatorname{eval}\left(t[e], G^{\prime}\right)=$ true if and only if $D P_{T}\left(G^{\prime} \cup \widetilde{E}\right)$ returns UNSATISFIABLE. 
Corollary 1. For a set of predicates $P$, if $t[e] \doteq S D P_{T}(P \cup \widetilde{P}, E)$, then for any $P^{\prime} \subseteq(P \cup \widetilde{P})$ representing a minterm over $P$ (i.e. $p_{i} \in P^{\prime}$ iff $\left.\neg p_{i} \notin P^{\prime}\right)$, $\operatorname{eval}\left(t[e], P^{\prime}\right)=\operatorname{eval}\left(\mathcal{F}_{P}(e), P^{\prime}\right)$.

Hence $t[e]$ is a shared expression for $\mathcal{F}_{P}(e)$, where $e$ denotes $\bigvee_{e_{i} \in E} e_{i}$. An explicit representation of $\mathcal{F}_{P}(e)$ can be obtained by first computing $t[e] \doteq$ $S D P_{T}(P \cup \widetilde{P}, E)$ and then enumerating the cubes over $P$ that make $t[e]$ true.

In the following sections, we will instantiate $T$ to be the EUF and DIF theories and show that $S D P_{T}$ exists for such theories. For each theory, we only need to determine the value of $\operatorname{maxDerivDepth}_{T}(G)$ for any set of predicates $G$.

Remark 1. It may be tempting to terminate the loop in step (2) of $\operatorname{SDP}_{T}(G, E)$ once the set of predicates in $W$ does not change across two iterations. However, this would lead to an incomplete procedure and the following example demonstrates this.

Example 1. Consider an example where $G$ contains a set of predicates that denotes an "almost" fully connected graph over vertices $x_{1}, \ldots, x_{n}$. G contains an equality predicate between every pair of variables except the edge between $x_{1}$ and $x_{n}$. Let $E \doteq\left\{x_{1}=x_{n}\right\}$.

After one iteration of the $S D P_{T}$ algorithm on this example, $W$ will contain an equality between every pair of variables including $x_{1}$ and $x_{n}$ since $x_{1}=x_{n}$ can be derived from $x_{1}=x_{i}, x_{i}=x_{n}$, for every $1<i<n$. Therefore, if the $S D P_{T}$ algorithm terminates once the set of predicates in $W$ terminates, the procedure will terminate after two steps.

Now, consider the subset $G^{\prime}=\left\{x_{1}=x_{2}, x_{2}=x_{3}, \ldots, x_{i}=x_{i+1}, \ldots, x_{n-1}=\right.$ $\left.x_{n}\right\}$ of $G$. For this subset of $G, D P_{T}\left(G^{\prime} \cup \widetilde{E}\right)$ requires $l g(n)>1$ (for $n>2$ ) steps to derive the fact $x_{1}=x_{n}$. Therefore $S_{D P}(G, E)$ does not simulate the action of $D P_{T}\left(G^{\prime} \cup \widetilde{E}\right)$. More formally, we can show that eval $\left(t[e], G^{\prime}\right)=$ false, but $G^{\prime} \cup \widetilde{E}$ is unsatisfiable.

\section{3 $S D P$ for Equality and Uninterpreted Functions}

The terms in this logic can either be variables or application of an uninterpreted function symbol to a list of terms. A predicate in this theory is $t_{1} \sim t_{2}$, where $t_{i}$ is a term and $\sim \in\{=, \neq\}$. For a set $G$ of EUF predicates, $G_{=}$and $G_{\neq}$denote the set of equality and disequality predicates in $G$, respectively. Figure 2 describes the inference rules for this theory.

Let $\operatorname{terms}(\phi)$ denote the set of syntactically distinct terms in an expression (a term or a formula) $\phi$. For example, terms $(f(h(x)))$ is $\{x, h(x), f(h(x))\}$. For a set of predicates $G$, terms $(G)$ denotes the union of the set of terms in any $g \in G$.

A decision procedure for EUF can be obtained by the congruence closure algorithm [17, described in Figure 5.

For a set of predicates $G$, let $m=|\operatorname{terms}(G)|$. We can show that if we iterate the loop in step (2) of $D P_{T}(G)$ (shown in Figure 3) for at least $3 m$ steps, then $D P_{T}$ can implement the congruence closure algorithm. More precisely, for two 
1. Partition the set of terms in $\operatorname{terms}(G)$ into equivalence classes using the $G=$ predicates. At any point in the algorithm, let $E C(t)$ denote the equivalence class for any term $t \in \operatorname{terms}(G)$.

(a) Initially, each term belongs to its own distinct equivalence class.

(b) We define a procedure merge $\left(t_{1}, t_{2}\right)$ that takes two terms as inputs. The procedure first merges the equivalence classes of $t_{1}$ and $t_{2}$. If there are two terms $s_{1} \doteq f\left(u_{1}, \ldots, u_{n}\right)$ and $s_{2} \doteq f\left(v_{1}, \ldots, v_{n}\right)$ such that $E C\left(u_{i}\right)=E C\left(v_{i}\right)$, for every $1 \leq i \leq n$, then it recursively calls merge $\left(s_{1}, s_{2}\right)$.

(c) For each $t_{1}=t_{2} \in G_{=}$, call merge $\left(t_{1}, t_{2}\right)$.

2. If there exists a predicate $t_{1} \neq t_{2}$ in $G_{\neq}$, such that $E C\left(t_{1}\right)=E C\left(t_{2}\right)$, then return UNSATISFIABLE; else SATISFIABLE.

Fig. 5. Simple description of the congruence closure algorithm

terms $t_{1}$ and $t_{2}$ in terms $(G)$, the predicate $t_{1}=t_{2}$ will be derived within $3 m$ iterations of the loop in step 2 of $D P_{T}(G)$ if and only if $E C\left(t_{1}\right)=E C\left(t_{2}\right)$ after step (1) of the congruence closure algorithm (the proof can be found in 12]).

Proposition 2. For a set of EUF predicates $G$, if $m \doteq|\operatorname{terms}(G)|$, then the value of maxDerivDepth ${ }_{T}(G)$ for the theory is bound by $3 \mathrm{~m}$.

Complexity of $S D P_{T}$. The run time and size of expression generated by $S D P_{T}$ depend both on $\operatorname{maxDerivDepth}_{T}(G)$ for the theory and also on the maximum number of predicates in $W$ at any point during the algorithm. The maximum number of predicates in $W$ can be at most $m(m-1) / 2$, considering equality between every pair of term. The disequalities are never used except for generating contradictions. It is also easy to verify that the size of $S(g)$ (used in step (2) of $S D P_{T}$ ) is polynomial in the size of input.Hence the run time of $S D P_{T}$ for EUF and the size of the shared expression returned by the procedure is polynomial in the size of the input.

\subsection{SDP for Difference Logic}

Difference logic is a simple yet useful fragment of linear arithmetic, where predicates are of the form $x \bowtie y+c$, where $x, y$ are variables, $\bowtie \in\{<, \leq\}$ and $c$ is a real constant. Any equality $x=y+c$ is represented as a conjunction of $x \leq y+c$ and $y \leq x-c$. The variables $x$ and $y$ are interpreted over real numbers. The function symbol "+" and the predicate symbols $\{<, \leq\}$ are the interpreted symbols of this theory. Figure 6 presents the inference rules for this theory 3 .

Given a set $G$ of difference logic predicates, we can construct a graph where the vertices of the graph are the variables in $G$ and there is a directed edge in the graph from $x$ to $y$, labeled with $(\bowtie, c)$ if $x \bowtie y+c \in G$. We will use a predicate and an edge interchangeably in this section.

Definition 4. A simple cycle $x_{1} \bowtie x_{2}+c_{1}, x_{2} \bowtie x_{3}+c_{2}, \ldots, x_{n} \bowtie x_{1}+c_{n}$ (where each $x_{i}$ is distinct) is "illegal" if the sum of the edges is $d=\Sigma_{i \in[1, n]} c_{i}$

$\overline{{ }^{3} \text { Constraints }}$ like $x \bowtie c$ are handled by adding a special variable $x_{0}$ to denote the constant 0 , and rewriting the constraint as $x \bowtie x_{0}+c$ [19]. 
and either (i) all the edges in the cycle are $\leq$ edges and $d<0$, or (ii) at least one edge is an $<$ edge and $d \leq 0$.

It is well known [6] that a set of difference predicates $G$ is unsatisfiable if and only the graph constructed from the predicates has a simple illegal cycle. Alternately, if we add an edge $(\bowtie, c)$ between $x$ and $y$ for every simple path from $x$ to $y$ of weight $c(\bowtie$ determined by the labels of the edges in the path), then we only need to check for simple cycles of length two in the resultant graph. This corresponds to the rules (C) and (D) in Figure 6.

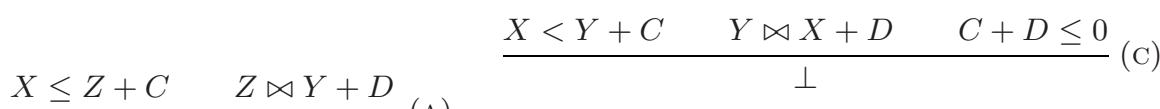

$$
\begin{aligned}
& \frac{X \leq Y+C \quad Y \leq X+D \quad C+D<0}{\perp} \\
& \frac{X \leq Y \quad Y \leq X}{X=Y}
\end{aligned}
$$

Fig. 6. Inference rules for Difference logic

For a set of predicates $G$, a predicate corresponding to a simple path in the graph of $G$ can be derived within $l g(m)$ iterations of step (2) of $D P_{T}$ procedure, where $m$ is the number of variables in $G$ (the proof is in [12]).

Proposition 3. For a set of DIF predicates $G$, if $m$ is the number of variables in $G$, then maxDerivDepth ${ }_{T}(G)$ for the DIF theory is bound by $\lg (m)$.

Complexity of $S D P_{T}$. Let $c_{\max }$ be the absolute value of the largest constant in the set $G$. We can ignore any derived predicate in of the form $x \bowtie y+C$ from the set $W$ where the absolute value of $C$ is greater than $(m-1) * c_{\max }$. This is because the maximum weight of any simple path between $x$ and $y$ can be at most $(m-1) * c_{\max }$. Again, let $\operatorname{const}(g)$ be the absolute value of the constant in a predicate $g$. The maximum weight on any simple path has to be a combination of these weights. Thus, the absolute value of the constant is bound by:

$$
C \leq \min \left\{(m-1) * c_{\max }, \Sigma_{g \in G} \operatorname{const}(g)\right\}
$$

The maximum number of derived predicates in $W$ can be $2 * m^{2} *(2 * C+1)$, where a predicate can be either $\leq$ or $<$, with $\mathrm{m}^{2}$ possible variable pairs and the absolute value of the constant is bound by $C$. This is a pseudo polynomial bound as it depends on the value of the constants in the input.

However, many program verification queries use a subset of difference logic where each predicate is of the form $x \bowtie y$ or $x \bowtie c$. For this case, the maximum number of predicates generated can be $2 * m *(m-1+k)$, where $k$ is the number of different constants in the input. 


\section{Combining $S D P$ for Saturation Theories}

In this section, we provide a method to construct a symbolic decision procedure for the combination of saturation theories $T_{1}$ and $T_{2}$, given $S D P$ for $T_{1}$ and $T_{2}$. The combination is based on an extension of the Nelson-Oppen (N-O) framework [16] that constructs a decision procedure for the theory $T_{1} \cup T_{2}$ using the decision procedures of $T_{1}$ and $T_{2}$.

We assume that the theories $T_{1}$ and $T_{2}$ have disjoint signatures (i.e., they do not share any function symbol), and each theory $T_{i}$ is convex and stably infinite 4 . Let us briefly explain the $\mathrm{N}-\mathrm{O}$ method for combining decision procedures before explaining the method for combining $S D P$.

\subsection{Nelson-Oppen Method for Combining Decision Procedures}

Given two theories $T_{1}$ and $T_{2}$, and the decision procedures $D P_{T_{1}}$ and $D P_{T_{2}}$, the N-O framework constructs the decision procedure for $T_{1} \cup T_{2}$, denoted as $D P_{T_{1} \cup T_{2}}$.

To decide an input set $G$, the first step in the procedure is to purify $G$ into sets $G_{1}$ and $G_{2}$ such that $G_{i}$ only contains symbols from theory $T_{i}$ and $G$ is satisfiable if and only if $G_{1} \cup G_{2}$ is satisfiable. Consider a predicate $g \doteq p\left(t_{1}, \ldots, t_{n}\right)$ in $G$, where $p$ is a theory $T_{1}$ symbol. The predicate $g$ is purified to $g^{\prime}$ by replacing each subterm $t_{j}$ whose top-level symbol does not belong to $T_{1}$ with a fresh variable $w_{j}$. The expression $t_{j}$ is then purified to $t_{j}^{\prime}$ recursively. We add $g^{\prime}$ to $G_{1}$ and the binding predicate $w_{j}=t_{j}^{\prime}$ to the set $G_{2}$. We denote the latter as binding predicate because it binds the fresh variable $w_{j}$ to a term $t_{j}^{\prime}$.

Let $V_{s h}$ be the set of shared variables that appear in $G_{1} \cap G_{2}$. A set of equalities $\Delta$ over variables in $V_{s h}$ is maintained; $\Delta$ records the set of equalities implied by the facts from either theory. Initially, $\Delta=\{\}$.

Each theory $T_{i}$ then alternately decides if $D P_{T_{i}}\left(G_{i} \cup \Delta\right)$ is unsatisfiable. If any theory reports UNSATISFIABLE, the algorithm returns UNSATISFIABLE; otherwise, the theory $T_{i}$ generates the new set of equalities over $V_{s h}$ that are implied by $G_{i} \cup \Delta_{5}^{5}$. These equalities are added to $\Delta$ and are communicated to the other theory. This process is continued until the set $\Delta$ does not change. In this case, the method returns SATISFIABLE. Let us denote this algorithm as $D P_{T_{1} \cup T_{2}}$.

Theorem 2 ([16]). For convex, stably infinite and signature-disjoint theories $T_{1}$ and $T_{2}, D P_{T_{1} \cup T_{2}}$ is a decision procedure for $T_{1} \cup T_{2}$.

There can be at most $\left|V_{s h}\right|$ irredundant equalities over $V_{s h}$, therefore the N-O loop terminates after $\left|V_{s h}\right|$ iterations for any input.

\subsection{Combining SDP Using Nelson-Oppen Method}

We will briefly describe a method to construct the $S D P_{T_{1} \cup T_{2}}$ by combining $S D P_{T_{1}}$ and $S D P_{T_{2}}$. As before, the input to the method is the pair $(G, E)$ and

\footnotetext{
${ }^{4}$ We need these restrictions only to exploit the $\mathrm{N}-\mathrm{O}$ combination result. The definition of convexity and stably infiniteness can be found in [16.

${ }^{5}$ We assume that each theory has an inference rule for deriving equality between variables in the theory, and $D P_{T}$ also returns a set of equality over variables.
} 
the output is an expression $t[e]$. The facts in $E$ are also purified into sets $E_{1}$ and $E_{2}$ and the new binding predicates are added to either $G_{1}$ or $G_{2}$.

Our goal is to symbolically encode the runs of the N-O procedure for $G^{\prime} \cup$ $\widetilde{E}$, for every $G^{\prime} \subseteq G$. For any equality predicate $\delta$ over $V_{s h}$, we maintain an expression $\psi_{\delta}$ that records all the different ways to derive $\delta$ (initialized to false). We also maintain an expression $\psi_{e}$ to record all the derivations of $e$ (initialized to false).

The N-O loop operates just like the case for constructing $D P_{T_{1} \cup T_{2}}$. The $S D P_{T_{i}}$ for each theory $T_{i}$ now takes $\left(G_{i} \cup \Delta, E_{i}\right)$ as input, where $\Delta$ is the set of equalities over $V_{s h}$ derived so far. In addition to computing the (shared) expression $t[e]$ as before, $S D P_{T_{i}}$ also returns the expression $t[(\delta, \top)]$, for each equality $\delta$ over $V_{s h}$ that can be derived in step (2) of the $S D P_{T}$ algorithm.

The leaves of the expressions $t[e]$ and $t[(\delta, \top)]$ are $G_{i} \cup \Delta$ (since leaves for $\widetilde{E_{i}}$ are replaced with true). We substitute the leaves for any $\delta \in \Delta$ with the expression $\psi_{\delta}$, to incorporate the derivations of $\delta$ until this point. We also update $\psi_{\delta} \leftarrow\left(\psi_{\delta} \vee t[(\delta, \top)]\right)$ to add the new derivations of $\delta$. Similarly, we update $\psi_{e} \leftarrow\left(\psi_{e} \vee t[e]\right)$ with the new derivations.

The N-O loop iterates $\left|V_{s h}\right|$ number of times to ensure that it has seen every derivation of a shared equality over $V_{s h}$ from any set $G_{1}^{\prime} \cup G_{2}^{\prime} \cup \widetilde{E_{1}} \cup \widetilde{E_{2}}$, where $G_{i}^{\prime} \subseteq G_{i}$

After the N-O iteration terminates, $\psi_{e}$ contains all the derivations of $e$ from $G$. However, at this point, there are two kind of predicates in the leaves of $\psi_{e}$; the purified predicates and the binding predicates. If $g^{\prime}$ was the purified form of a predicate $g \in G$, we replace the leaf for $g^{\prime}$ with $b_{g}$. The leaves of the binding predicates are replaced with true, as the fresh variables in these predicates are really names for subterms in any predicate, and thus their presence does not affect the satisfiability of a formula. Let $t[e]$ denote the final expression for $\psi_{e}$ that is returned by $S D P_{T_{1} \cup T_{2}}$. Observe that the leaves of $t[e]$ are variables in $B_{G}$.

Theorem 3. For two convex, stably-infinite and signature-disjoint theories $T_{1}$ and $T_{2}$, if $t[e] \doteq S D P_{T_{1} \cup T_{2}}(G, E)$, then for any set of predicates $G^{\prime} \subseteq G$, $\operatorname{eval}\left(t[e], G^{\prime}\right)=$ true if and only if $D P_{T_{1} \cup T_{2}}\left(G^{\prime} \cup \widetilde{E}\right)$ returns UNSATISFIABLE.

Since the theory of EUF and DIF satisfy all the restrictions of the theories of this section, we can construct an $S D P$ for the combined theory that still runs in pseudo-polynomial time.

\section{Implementation and Results}

We have implemented a prototype of the symbolic decision procedure for the combination of EUF and DIF theories. To construct $\mathcal{F}_{P}(e)$, we first build a BDD (using the CUDD [7] BDD package) for the expression $t[e]$ (returned by $\left.S D P_{T}(P \cup \widetilde{P}, E)\right)$ and then enumerate the cubes from the BDD.

Creating the BDD for the shared expression $t[e]$ and enumerating the cubes from the BDD can have exponential complexity in the worst case. This is because 


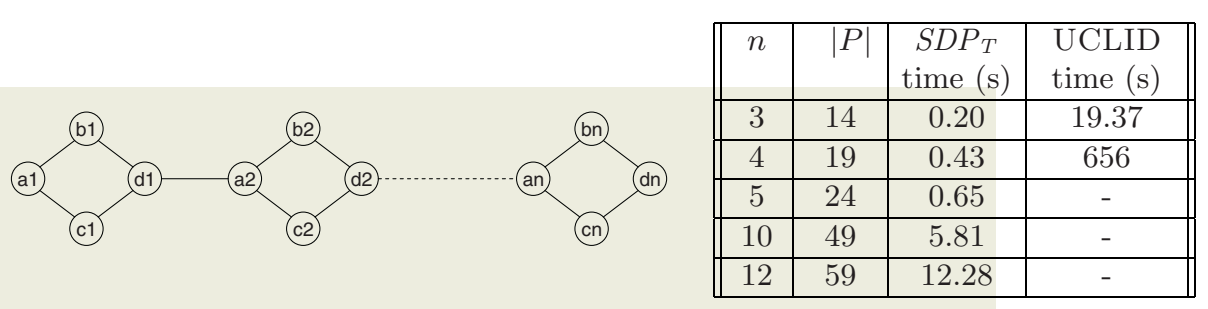

Fig. 7. Result on diamond examples with increasing number of diamonds. The expression $e$ is $(a 1=d n)$. A "-" denotes a timeout of 1000 seconds

the expression for $\mathcal{F}_{P}(e)$ can involve an exponential number of cubes (e.g. the example in Fig 7). However, most problems in practice have a few cubes in $\mathcal{F}_{P}(e)$. Secondly, as the number of leaves of $t[e]$ (alternately, number of BDD variables) is bound by $|P|$, the size of the overall BDD is usually small, and is computed efficiently in practice. Finally, by generating only the prime implicant 6 of $\mathcal{F}_{P}(e)$ from the BDD, we obtain a compact representation of $\mathcal{F}_{P}(e)$.

We report preliminary results evaluating our symbolic decision procedure based predicate abstraction method on a set of software verification benchmarks. The benchmarks are generated from the predicate abstraction step for constructing Boolean Programs from C programs of Microsoft Windows device drivers in SLAM [2].

We compare our method with two other methods for performing predicate abstraction: (i) DP-based: This method uses the decision procedure ZAPATO [1] to enumerate the set of cubes that imply $e$. Various optimizations (e.g. considering cubes in increasing order of size) are used to prevent enumerating exponential number of cubes in practice. (ii) UCLID-based: This method performs quantifier-elimination using incremental SAT-based methods [13.

To compare with the DP-based method, we generated 665 predicate abstraction queries from the verification of device-driver programs. Most of these queries had between 5 and 14 predicates in them and are fairly representative of queries in SLAM. The run time of DP-based method was 27904 seconds on a $3 \mathrm{GHz}$. machine with 1 GB memory. The run time of SDP-based method was 273 seconds. This gives a little more than 100X speedup on these examples, demonstrating that our approach can scale much better than decision procedure based methods. We have not been able to run UCLID-based method on SLAM benchmarks at the point of submitting this paper.

To compare with UCLID-based approach, we generated different instances of a problem (see Figure 7 for the example) where $P$ is a set of equality predicates representing $n$ diamonds connected in a chain and $e$ is an equality $a 1=d n$. We generated different problem instances by varying the size of $n$. For an instance

${ }^{6}$ For any Boolean formula $\phi$ over variables in $V$, prime implicants of $\phi$ is a set of cubes $C \doteq\left\{c_{1}, \ldots, c_{m}\right\}$ over $V$ such that $\phi \Leftrightarrow \bigvee_{c \in C} c$ and two or more cubes from $C$ can't be combined to form a larger cube. 
with $n$ diamonds, there are $5 n-1$ predicates in $P$ and $2^{n}$ cubes in $\mathcal{F}_{P}(e)$ to denote all the paths from $a 1$ to $d n$. Figure 7 shows the result comparing both the methods. We should note that UCLID method was run on a slightly slower $2 \mathrm{GHz}$ machine. The results illustrate that our method scales much better than the SATbased enumeration used in UCLID for this example. Intuitively, UCLID-based approach grows exponentially with the number of predicates $\left(2^{|P|}\right)$, whereas our approach only grows exponentially with the number of diamonds $\left(2^{n}\right)$ in the result.

\section{References}

1. T. Ball, B. Cook, S. K. Lahiri, and L. Zhang. Zapato: Automatic Theorem Proving for Software Predicate Abstraction Refinement. In Computer Aided Verification ( $C A V^{\prime}$ '04), LNCS 3114. Springer-Verlag, 2004.

2. T. Ball, R. Majumdar, T. Millstein, and S. K. Rajamani. Automatic predicate abstraction of $\mathrm{C}$ programs. In Programming Language Design and Implementation (PLDI '01), Snowbird, Utah, June, 2001. SIGPLAN Notices, 36(5), May 2001.

3. R. E. Bryant. Graph-based algorithms for Boolean function manipulation. IEEE Transactions on Computers, C-35(8), August 1986.

4. S. Chaki, E. M. Clarke, A. Groce, S. Jha, and H. Veith. Modular Verification of Software Components in C. IEEE Transactions on Software Engineering, 30(6), June 2004.

5. E. Clarke, D. Kroening, N. Sharygina, and K. Yorav. Predicate abstraction of ANSI-C programs using SAT. Formal Methods in System Design (FMSD), 25, 2004.

6. T. H. Cormen, C. E. Leiserson, and R. L. Rivest. Introduction to Algorithms. MIT Press, 1990.

7. CUDD:CU Decision Diagram Package. Available at http://vlsi.colorado. edu/ fabio/CUDD/cuddIntro.html.

8. S. Das, D. Dill, and S. Park. Experience with predicate abstraction. In ComputerAided Verification (CAV '99), LNCS 1633. Springer-Verlag, July 1999.

9. C. Flanagan and S. Qadeer. Predicate abstraction for software verification. In Symposium on Principles of programming languages (POPL '02). ACM Press, 2002.

10. S. Graf and H. Saïdi. Construction of abstract state graphs with PVS. In ComputerAided Verification (CAV' '97), LNCS 1254. Springer-Verlag, June 1997.

11. T. A. Henzinger, R. Jhala, R. Majumdar, and G. Sutre. Lazy Abstraction. In Symposium on Principles of programming languages (POPL '02). ACM Press, 2002.

12. S. K. Lahiri, T. Ball, and B. Cook. Predicate abstraction via symbolic decision procedures. Technical Report MSR-TR-2005-53, Microsoft Research, April 2005.

13. S. K. Lahiri, R. E. Bryant, and B. Cook. A symbolic approach to predicate abstraction. In Computer-Aided Verification (CAV 2003), LNCS 2725. Springer-Verlag, 2003.

14. K. McMillan. Applying SAT Methods in Unbounded Symbolic Model Checking. In Proc. Computer-Aided Verification (CAV'02), LNCS 2404, July 2002.

15. K. S. Namjoshi and R. P. Kurshan. Syntactic program transformations for automatic abstraction. In Computer Aided Verification, LNCS 1855, 2000.

16. G. Nelson and D. C. Oppen. Simplification by cooperating decision procedures. ACM Transactions on Programming Languages and Systems (TOPLAS), 2(1), 1979. 
17. G. Nelson and D. C. Oppen. Fast decision procedures based on the congruence closure. Journal of the ACM, 27(2), 1980.

18. H. Saïdi and N. Shankar. Abstract and model check while you prove. In ComputerAided Verification, volume 1633 of LNCS. Springer-Verlag, July 1999.

19. O. Strichman, S. A. Seshia, and R. E. Bryant. Deciding Separation Formulas with SAT. In Proc. Computer-Aided Verification (CAV'O2), LNCS 2404, July 2002. 